Untersuchungen, am besten an höheren Säugetieren (Katze, Hund), zu zeigen, daß auch bei diesen ähnliche Beziehungen zwischen Wach- und Schlafzustand auf der einen und dem bioelektrischen Zustandsbild der Rinde auf der anderen Seite vorhanden sind wie beim Menschen und wie sie beim Kaninchen vorzuliegen scheinen.

Gewisse Anhaltspunkte für das Vorhandensein derartiger Beziehungen liefern übrigens die hirnbioelektrischen Untersuchungen von R. Klaue über den Wachund Schlafzustand der Katze. Nach Kla u e ${ }^{21}$ ist bei dieser der Schlafzustand (oder genauer der nicht sehr tiefe Schlaf) hirnbioelektrisch charakterisiert durch das Auftreten von unregelmäßigen trägen Schwankungen, die an Amplitude wesentlich größer sind als die erheblich regelmäßigeren Wellen des Wachzustandes. Außerdem traten (besonders deutlich im künstlichen Schlaf durch Veronal, aber auch im natürlichen Schlaf) in unregelmäßigen Abständen Gruppen regelmäßiger Schwankungen von etwa $12 \mathrm{~Hz}$ auf. Doch wäre es erwünscht, daß diese Untersuchungen mit einer verbesserten Versuchstechnik wiederholt würden.

Sollte die hier geäußerte Vermutung über die Bedeutung der 6-Hz-Schwankungen und der Feld- eigenströme zutreffen, so müßte damit die Frage nach der Existenz von „Feldeigenströmen“, also von Potentialschwankungen, die eine für jedes architektonische Feld der Rinde charakteristische Ablaufsform besitzen, aufs neue eingehend geprüft werden. Ferner aber wäre im Zusammenhang mit der Tatsache, daß die 6-Hz-Schwankungen auch von subcortikalen Gebieten abgeleitet werden konnten, ein Weg zur experimentellen Beantwortung der Frage nach dem Ursprung der für den Wachzustand charakteristischen Potentialschwankungen (also beim Menschen der $\alpha$-Wellen) gewiesen. Damit würde vielleicht auch ein neues Licht auf jenen verborgenen Mechanismus fallen, der den Übergang vom Wach- in den Schlafzustand und umgekehrt regelt. Daß dabei im $\mathrm{Zu}$ sammenhang mit gleichzeitigen Ableitungen von der Rinde und von subcortikalen Gebieten u. a. auch die Methoden der Reizung und Ausschaltung subcortikaler Hirnabschnitte anzuwenden sein werden, braucht kaum besonders betont zu werden.

21 J. Psychol. Neurol. 47, 510 [1937].

\title{
Das einstige und heutige Vorkommen der wilden Weinrebe im Oberrheingebiet
}

Von Franz KirchHeimer

(Z. Naturforschg. 1, 410-413 [1946]; aus Freiburg i. Br. eingegangen am 22. Mai 1946)

Aus einer der Zukunft vorbehaltenen größeren Darstellung werden die heutigen und früheren Standorte der im Oberrheingebiet urwüchsigen Vitis silvestris mitgeteilt. Die Verwüstung der stromnahen Wälder durch den Krieg läßt befürchten, daß die wilden Weinreben überwiegend den Untergang fanden und die folgenden Angaben lediglich als èin Nachruf auf das einstige Vorkommen eines der bemerkenswertesten Gewächse der mitteleuropäischen Flora zu bewerten sind.

$\mathrm{M}$ it dem Auftreten der als Vitis silvestris bekannten wilden Weinrebe im Oberrheintal habe ich mich unter besonderer. Berücksichtigung der nördlichsten Standorte beschäftigt ${ }^{1}$. Inzwischen wurde die Gesamtheit ihrer früheren und gegenwärtigen Vorkommen zu erfassen versucht. Die Grundlage dieser Erhebung bildete eine Rundfrage bei sämtlichen Forstämtern des Gebietes. Begehungen der dem Rheine benachbarten Wälder dienten zur Prüfung der erhaltenen Angaben und sonstiger Nachrichten über die Standorte der wilden Weinrebe.

1 Vergl. Wein u. Rebe 46, 15-22 [1944]; Der Deutsche Weinbau 23, 207—209 [1944]. In diesen Darstellungen sind die wichtigsten Schriften über Vitis silvestris und ihre nördlichen Vorkommen nachgewiesen.
Den Ablauf dieser Untersuchungen haben die Zeitverhältnisse erheblich beeinträchtigt. Jedoch mußten sie fortgesetzt werden, da die kriegerischen Ereignisse im Oberrheintal und die mit ihnen verbundenen Wehrbauten zu Zweifeln an der weiteren Erhaltung der Vitis silvestris veranlaßten. Schwierig hat sich die Erfassung der früheren Standorte nach dem Schrifttum und älteren Akten der Forstbehörden gestaltet. Das für die Durchsicht vorgesehene Material konnte nicht vollständig beschafft werden.

Ein Vorkommen wilder Weinreben in Mitteleuropa wurde früher von den meisten Autoren bestritten oder angezweifelt. Man hat das als Vitis silvestris Gmelin beschriebene Gewächs für eine verwilderte Form der 
unter V.vinifera L. vereinigten Kulturreben gehalten, obwohl sich eine erhebliche. Verschiedenheit erkennen läßt. Der als funktionelle-Diözie beschriebenen Erscheinung begegnet man nur bei den wilden Reben. Lediglich vereinzelt ist die den angebauten Formen in der Regel eigene Zwitterblütigkeit anzutreffen. Diesem für die Vitis silvestris bezeichnenden geschlechtlichen Unterschied entspricht ein deutlicher Dimorphismus der vegetativen Organe. Blätter der männlich blühenden Pflanzen erscheinen gegenüber dem fast ungelappten Laub der weiblichen Stöcke \pm eingeschnitten. Ferner sind die wilden Weinreben in den nördlichen Teilen des Areals fast nur Waldbewohner und bevorzugen feuchte Standorte, besonders die an den größeren Flußläufen gelegenen Auen. Die Samen der angepflanzten Reben werden zwar nicht selten an solche Stellen verschleppt, haben aber niemals zu einem Verwildern ihrer Formen geführt. In der Kultur erwiesen sich die Merkmale der Vitis silvestris als beständig. Auch ist festgestellt, da $\$$ unter keinen Bedingungen eine Konvergenz der angepflanzten Reben nach der fast stets blaufrüchtigen wilden Art erreicht wird. Selbst die viele Jahrzehnte ,verwilderten“ Stöcke aufgelassener Kulturen bewahren die 'der betreffenden Sorte eigenen Merkmale, so daß sie unschwer von Vitis silvestris zu unterscheiden sind.

Diese Befunde beseitigen die Zweifel an der Urwüchsigkeit der Weinrebe im Gebiet der mitteleuropäischen Flora. Trotzdem erteilen selbst neuzeitliche Lehr- und Handbücher der Botanik keinen Hinweis auf ihr Vorkommen. Das Verhältnis der Vitis silvestris zu den Kulturreben wird verschieden beurteilt. Gmelin ${ }^{2}$ hat die wilde Weinrebe für eine besondere Art gehalten. Andere Autoren betrachten sie lediglich als spontane „Varietät" der Vitis vinifera und auch vom Mansfeldschen Verzeichnis wird das Gewächs in diesem Sinne geführt ${ }^{3}$. Jedoch hat „Vitis vinifera" zunächst lediglich die angebauten Reben bezeichnet. Man ist aber nicht berechtigt, eine Pflanze erwiesener Urwüchsigkeit in den nur für Kulturformen geprägten Namen einzuschließen. Nach dieser Sachlage kann für die Wildreben der mitteleuropäischen Flora nur die Gmelin sche Bezeichnung Vitis silvestris verwenđet werden. Ein Teil der im Gebiet angepflanzten Sorten dürfte von heimischen Rassen dieser Art abstammen oder sie eingekreuzt enthalten. Andere Kulturreben erinnern an einige der im Mittelmeerbereich und Kaukasus vorkommenden Wildreben, ohne daß ihr näheres Verhältnis zu diesen Formen der Vitis silvestris bestimmt werden kann.

Ungeachtet der zeitbedingten Lücken ist eine Ubersicht der im Oberrheingebiet festgestellten einstigen Standorte von Vitis silvestris wichtig. Mit der Bedeutung des heutigen Vorkommens und seiner nunmehr durchgeführten Erfassung hat sich meine erwähnte Schrift beschäftigt. Wahrscheinlich ist die Mehrzahl der in den Jahren 1943 und 1944 festgestellten Stöcke dem Kriege oder seinen Folgen zum Opfer gefallen. Nur der völlige Schutz

2 Flora badenisis I, 543-545 [1806].

3 Verzeichnis d. Farn- u. Blütenpflanzen des Deutschen Reiches, S. 166 [Jena 1940]. kann die drohende Gefahr des Verschwindens der wilden Weinrebe aus dem Oberrheintal bannen. Nähere Einzelheiten über die Standorte der Vitis silvestris und ihr früheres Vorkommen bleiben einer späteren Darstellung größeren. Umfanges vorbehalten. Sie wird sich auch mit der Beschaffenheit der Früchte und den für Fragen des Weinbaus wichtigen Feststellungen zu beschäftigen haben.

I. Gegenwärtiges Vorkommen nach den Angaben der Forstämter und eigenen Erhebungen

Die wichtigsten Standorte der Vitis silvestris finden sich auf der Rheininsel unweit Ketsch, im Stadtwald von Mannheim und bei Hördt. An diesen Stellen wurden neben den überwiegenden männlichen Stöcken einzelne weibliche und zwitterige Reben festgestellt. Sie reifen sämtlich blaue Früchte, deren Geschmack zwischen sauer und angenehm süßlich schwankt. Auch die seit mehreren Jahrzehnten bekannte Rebe im Waldpark Neckarau und ein bei Otterstadt gedeihender Stock entwickeln Trauben von der gleichen Farbe. Die Gesamtzahl der wilden Weinreben des Oberrheingebietes wurde gegen das Ende des Jahres 1944 auf weniger als 60 geschätzt. Der schnelle Rückgang des Bestandes während der jüngsten Vergangenheit ergibt sich besonders aus dem Vergleich der im Bezirk des Forstamtes Erstein wiederholten Zählungen, über die umfangreiche Akten berichten.

Bischweiler i. Elsaß (Gemeindewald Offendorf Abt. 12 a, ein Stock unweit der Pflanzschule).

Erstein i. Elsaß (ein Stock im Gemeindewald Matzenheim Abt. 12; zwei Stöcke im Gemeindewald Sermersheim Abt. 1 u. 3).

Germersheim i. d. Pfalz (zehn Stöcke im Staatswald Hördt Abt. IX u. XI).

Kolmar i. Elsaß (drei Stöcke im Gemeindewald Fronholz Abt. 34 u. 41, Neuland Abt. 17).

Ludwigshafen i. d. Pfalz (ein Stock im Stadtpark) ${ }^{4}$

Mannheim i. Baden (sieben Stöcke im Stadtpark auf der Reißinsel; ein Stock im Waldpark Neckarau).

Schwetzingen i. Baden (etwa zwanzig Stöcke im Dienstbezirk I, Rheinwald Distrikt III Abt. 8 u. 9, sog. Rheininsel b. Ketsch; ein Stock im Distrikt IV Abt. 8, sog. Kollerinsel b. Brühl).

Speyer i. d. Pfalz (vier Stöcke im Gemeindewald Otterstadt Abt. 5 u. 6, sog. Angelwald).

4 Fliegerbomben haben diese Rebe und einen Teil der für Mannheim erwähnten Stöcke erheblich beschädigt. 
II. Gegenwärtiges Vorkommen fraglich

An den Standorten der folgenden Liste hat sich die wilde Weinrebe ohne $Z_{\text {weifel vorgefunden. Ein }}$ Teil von ihnen ist im früheren Schrifttum erwähnt oder durch Herbarexemplare für vergangene Jahrzehnte belegt. Jedoch konnte die Vitis silvestris an diesen Stellen weder von den zuständigen Forstämtern noch durch meine Bemühungen gefunden werden. Für einige Standorte ist der Zeitpunkt des Absterbens bestimmter Stöcke bekannt. Immerhin besteht die Möglichkeit, daß in den schwer zugänglichen und durch ein dichtes Unterholz unübersichtlichen rheinnahen Wäldern auch gegenwärtig einzelne Reben ein verborgenes Dasein führen. Allerdings haben zahlreiche Nachfragen und sonstige Bemühungen keine Hinweise auf weitere Vorkommen der Vitis silvestris im Gebiet ergeben.

Breisach i. Baden (zwischen Jechtingen und Saßbach an der Sponeck bis 1939).

Germersheim i. d. Pfalz (Stadtwald auf der Insel Elisabethenwörth; nach Auskunft des zuständigen Forstamtes erloschen).

Graben i. Baden (Gemeindewald Linkenheim; ein Stock in der Abt. II 16 während des Winters 1941/1942 abgestorben).

Markolsheim i. Elsaß (Privatwald am Forsthaus Mauchen bis 1914).

Rheinau i. Elsaß (Auwald, Is sler 1938).

Rufach i. Elsaß (Thurwald, Is sler 1938).

Schlettstadt i. Elsaß (bei Sundhausen, Is sler 1938).

III. Vorkommen nach den Angaben des Schrifttums

Das folgende Verzeichnis erfaßt die als Standorte der wilden Weinrebe erwähnten Örtlichkeiten im Oberrheingebiet. Die Durchsicht des gegenwärtig nicht verfügbaren Schrifttums wird zu einer Erweiterung dieser Liste führen. Viele Standortsangaben beziehen sich lediglich auf die Stromebene oder größere Städte, z. B. selbst in der grundlegenden Darstellung Bronners aus dem Jahre 1857. Sie werden vom Verzeichnis zugunsten der genaueren Hinweise übergangen ${ }^{5}$.

Durch den Vergleich der zahlreichen Namen auf der folgenden Liste mit den wenigen Standorten der Gegenwart wird ein dem Aussterben sich nähernder Rückgang der Vitis silvestris während der letzten Jahrzehnte angezeigt ${ }^{6}$. Selbst seit 1943 sind z. B. die Vorkommen westlich von Darmstadt bei Erfelden und Groß-Gerau erloschen. Einige der

5 Die Standorte sind mit den Namen der Autoren und dem Jahr ihrer Mitteilung belegt. Der Nachweis dieser Schriften erfolgt in der angekündigten Abhandlung über die wilden Weinreben des Oberrheingebietes. erwähnten Standorte könnten nach ihrer Lage zu früheren oder heutigen Rebenpflanzungen als Kulturrelikte zu deuten sein. Diese Möglichkeit wird in meiner größeren Darstellung erörtert und für sie verneint.

a) Schweiz.

Baden a. d. Limmat (v. Haller 1742).

Brugg.a.d. Aare (Gesner 1561).

Münchenstein a. d. Birs (v. Haller 1742).

b) B a den.

Badenweiler b. Müllheim (,Bottenwäldchen“: Gmelin 1806).

Müllheim (rheinnahe Wälder: Spenner 1829; Döll 1862; Seubert 1875, 1880, 1891 u. 1905).

Neuenburg b. Müllheim (Döll 1862; Schildknecht 1863).

Sponeck a. Kaiserstuhl (O ber lin 1900; Jechtingen, Neuberger 1912; Sleumer 1934; Müller 1937 u. 1938; Bertsch 1939 u. 1940 Sasbach u. Jechtingen, Müller-Stoll 1941).

Rust b. Lahr (Schildknecht 1863).

Au b. Karlsruhe (Gmelin 1806).

Dachslanden b. Karlsruhe (Gmelin 1806; Döll 1862; K neucker 1886).

Knielingen b. Karlsruhe (Gmelin 1806; Döll 1862; Kneucker 1886; Oberlin 1900).

Eggenstein b. Karlsruhe (D öll1862; K n e u c k e r 1886).

Linkenheim b. Bruchsal (G melin 1806).

Neulußheim b. Wiesloch (D öll 1862).

Wiesloch (rheinnahe Wälder: Schmidt 1857; Döll 1862; Seubert 1875, 1880 u. 1891; Oberlin 1900; M üller 1930).

Ketsch b. Schwetzingen (Schultz 1846; Scheu 1937; K irch heimer 1939).

Schwetzingen (rheinnahe Wälder: v. Kettner 1815; Döll1862).

Heidelberg (Sümpfe am „Haarlaß“: D i e r ba ch 1819).

Neckarau b. Mannheim (Succow 1822; Oberlin 1900 u. 1907; v. Bassermann-Jordan 1923).

Mannheim (rheinnahe Wälder: Döll 1862 u. A.).

c) Frankreich (Elsaß).

Rülisheim b. Mülhausen (O b e r lin 1900).

Rufach b. Kolmar (Thurwald, Oberlin 1900; Issler 1938).

Kolmar (Blaeschweier, Fronholz, Neuland, Oberlin 1900; Fronholz, Iss le r 1924, 1926 und 1938).

Arzenheim b. Markolsheim (Oberlin 1900).

Sundhausen b. Schlettstadt (I s s le r 1938).

Müttersholz b. Schlettstadt (Krause 1913).

Diebolsheim b. Rheinau (O berlin 1900).

Friesenheim b. Rheinau (Issler 1938 nach Oberlin 1913).

Rheinau (Issler 1938 nach Oberlin 1913).

Sermersheim b. Erstein (Oberlin 1900; Wanner 1917; Issler 1938 nach Oberlin 1889 u. 1913).

- Die Mehrzahl der Angaben aus den früheren Jahrzehnten bezieht sich auf vor langer Zeit erloschene und zum Teil überbaute Standorte. 
Hüttenheim b. Erstein (Issler 1938 nach Oberlin 1889 u. 1913).

Bonfeld b. Erstein (Issler 1938 nach Oberlin 1913).

Matzenheim b. Erstein (Gemeindewald, Solms-L a u bach 1901; Issler 1938 nach Oberlin 1889 und 1913).

Osthausen b. Erstein (Gemeindewald, I s s le r 1938 nach Oberlin 1889 u. 1913).

Ostwald b. Illkirch (Mappus 1742; Gmelin 1806).

Ostwinkel b. Straßburg (M a p pus 1742; G m el in 1806).

Offendorf b. Bischweiler (Oberlin 1900).

Drusenheim b. Bischweiler (Oberlin 1900; K rause 1913).

d) Pfalz.

Wörth b. Landau (v. Bassermann-Jordan 1923).

Rohrbach b. Landau (W üst 1924).

Essingen b. Landau (Gemeindewald: v. Bas s e r m a n n Jordan 1923).

Neupfotz b. Germersheim (Morio 1921; v. Bas s e rman n - J ordan 1923).

Leimersheim b. Germersheim (G m elin 1806; B e r t s ch 1936).

Hördt b. Germersheim (v. B a s sermann-Jor da n 1923; Sch eu 1937; Staatswald, H e p p 1939).

Sondernheim b. Germersheim (v. B a s s e r m a n n - J o r dan 1907; Vollmann 1914; Morio 1921; v. Bassermann-Jordan 1923; Wilde 1935; Bertsch 1939 u. 1940; Kling 1941).

Germersheim (Vollmann 1914; Stadtwald, v. Bas sermann-Jordan 1923; Hegi 1923; Müller 1930; Werth 1931; Wilde 1935; Bertsch 1939 u. 1940; Elisabethenwörth, K ling 1941).

Lingenfeld b. Germersheim (Gemeindewald: v. Bassermann-Jordan 1923).

Harthausen b. Speyer (Schultz 1846).

Otterstadt b. Speyer (Gemeindewald, Schultz 1846; Hepp 1939).

Speyerdorf b. Neustadt a. d. Weinstraße (Bensenloch, v. Bassermann-Jordan 1907, Wilde 1918 und 1935; M ori o 1921; v. B a s s er ma n n-Jordan 1923).

Ludwigshafen (Stadtpark, Wilde 1935; B e r t s ch 1939 u. 1940).

e) Hessen.

Erfelden (Kühkopf, S e c r e t a m 1929; Kühkopf, Knoblochsaue, Schwedensäule, K i r c h h e i m e r 1944).

Dornberg b. Groß-Gerau (Fasanorie, Kirchheimer 1944).

Büttelborn b. Darmstadt (,Hecken“: D os ch u. Sc ri b a 1873, 1878 u. 1888; K i r ch heimer 1944).

Vilbel (Hexenberg: Gärtner, Meyer u. Scherbius 1799; Röhling 1812; Becker 1828; Fresenius 1932; Cassebeer u. Theobald 1849; Kirchheimer 1944).

\section{Vermeintliche Vorkommen}

Ein Teil der im Schrifttum wiederholten Angaben über Funde der Vitis silvestris bezieht sich ohne Zweifel auf die Reste früherer Kulturen, von denen einzelne Stöcke ihren Untergang überlebt haben. Dagegen ist mir aus dem Gebiet kein siche- rer Fall einer „Verwilderung“ der Edelreben durch verschleppten Samen bekannt. Einige Hinweise dürften Standorte der Vitis silvestris bezeichnen, obwohl sich die betreffenden Stöcke nicht in den stromnahen Wäldern fanden. FeuchteStellen der die Rheinebene säumenden Hügel haben ihr sehr ähnliche Lebensbedingungen geboten. So entsprechen die Einzelheiten eines zu Beginn des vergangenen Jahrhunderts bemerkten Vorkommens auf dem Schönberg unweit Freiburg wohl nur der wilden Form. Jedoch ist in diesem Fall und bei ähnlichen Siandorten, z. B. oberhalb Wiesloch, kein Beweis für die Urwüchsigkeit zu erbringen, da die Reben von den Hügeln des Gebietes längst verschwunden sind. Mit Sicherheit wurden lediglich die folgenden Vorkommen als Reste eines früheren Weinbaues erkannt:

Überlingen i. Baden (Hügel zwischen Goldbach und Sipplingen: Hö fl e 1850; S e u b e r t 1875, 1880, 1891 u. 1905).

Dossenheim b. Heidelberg i. Baden (Oberlin 1900; Scheu 1937).

Niederaspach i. Elsaß (Oberlin 1900).

Mutzig i. Elsaß (Dreispitz, K r a u s e 1913).

Dieuze i. Lothringen (O berlin 1900; H e g i 1923 u. A.). Leistadt b. Bad Dürkheim i. d. Pfalz (Z i mmermann 1907).

Bad Kreuznach (König 1843).

Auch die Umfrage nach dem Vorkommen der wilden Weinrebe wurde von einigen Forstämtern mit Hinweisen auf Kulturrelikte beantwortet:

Hohentwiel b. Singen (Württemberg).

Kadelburg b. Waldshut (Baden).

Bombach b. Kenzingen (Baden).

Ehrenstetten b. Staufen (Baden).

Pfirt (Elsaß).

Nach einer von mir veranlaßten Erhebung durch die Oberforstämter der Kantone des Rheingebietes finden sich in der Schweiz keine wilden Weinreben, sondern nur Reste aufgelassener Kulturen. Auch die Stöcke an einzelnen Hängen südlich von Basel dürften entgegen einer gelegentlich geäußerten Meinung diese Herkunft besitzen. Selbst die alten Angaben über Vorkommen bei Baden, Brugg und Münchenstein sind nicht mit Notwendigkeit auf die Vitis silvestris $\mathrm{zu}$ beziehen. Jedoch ist ihre einstige Urwüchsigkeit an diesen Örtlichkeiten wahrscheinlich, da die sie berührenden Flußläufe von Auwäldern begleitet'wurden. Ferner lassen die Erklärungen eines Glossars des Klosters Reichenau aus dem 13. Jahrhundert ein bis in das Bodenseegebiet ausgedehntes früheres Vorkommen der wilden Weinrebe vermuten. 